\title{
WEIGHTED PLANCHEREL ESTIMATES AND SHARP SPECTRAL MULTIPLIERS FOR THE GRUSHIN OPERATORS
}

\author{
Alessio Martini And Adam Sikora
}

Abstract. We study the Grushin operators acting on $\mathbb{R}_{x^{\prime}}^{d_{1}} \times \mathbb{R}_{x^{\prime \prime}}^{d_{2}}$ and defined by the formula

$$
L=-\sum_{j=1}^{d_{1}} \partial_{x_{j}^{\prime}}^{2}-\left(\sum_{j=1}^{d_{1}}\left|x_{j}^{\prime}\right|^{2}\right) \sum_{k=1}^{d_{2}} \partial_{x_{k}^{\prime \prime}}^{2}
$$

We obtain weighted Plancherel estimates for the considered operators. As a consequence we prove $L^{p}$ spectral multiplier results and Bochner-Riesz summability for the Grushin operators. These results are sharp if $d_{1} \geq d_{2}$. We discuss also an interesting phenomenon for weighted Plancherel estimates for $d_{1}<d_{2}$. The described spectral multiplier theorem is the analogue of the result for the sublaplacian on the Heisenberg group obtained by Müller and Stein and by Hebisch.

\section{Introduction}

Let $(\mathrm{X}, \mu)$ be a measure space and $L$ be a (possibly unbounded) self-adjoint operator on $L^{2}(\mathrm{X})$. If $E$ denotes the spectral resolution of $L$ on $\mathbb{R}$, then a functional calculus for $L$ can be defined via spectral integration and, for every Borel function $F: \mathbb{R} \rightarrow \mathbb{C}$, the operator

$$
F(L)=\int_{\mathbb{R}} F(\lambda) d E(\lambda)
$$

is bounded on $L^{2}(\mathrm{X})$ if and only if the "spectral multiplier" $F$ is an (E-essentially) bounded function. Characterizing, or at least giving (non-trivial) sufficient conditions for the $L^{p}$-boundedness of the operator $F(L)$, for some $p \neq 2$, in terms of properties of the multiplier $F$, is a much more complicated issue, and a huge amount of literature is devoted to instances of this problem (we refer the reader to $[4,6,10,11,13,14,18$, $21,24,26]$ for a detailed discussion of the relevant literature).

Here we are interested in the case where $\mathrm{X}=\mathbb{R}^{d_{1}} \times \mathbb{R}^{d_{2}}$, with Lebesgue measure, and $L$ is the Grushin operator, that is,

$$
L=-\Delta_{x^{\prime}}-\left|x^{\prime}\right|^{2} \Delta_{x^{\prime \prime}},
$$

where $x^{\prime}, x^{\prime \prime}$ denote the two components of a point $x \in \mathbb{R}^{d_{1}} \times \mathbb{R}^{d_{2}}$, while $\Delta_{x^{\prime}}, \Delta_{x^{\prime \prime}}$ are the corresponding partial Laplacians, and $\left|x^{\prime}\right|$ is the Euclidean norm of $x^{\prime}$.

Received by the editors May 24, 2012. 
Let $W_{q}^{s}(\mathbb{R})$ denote the $L^{q}$ Sobolev space on $\mathbb{R}$ of (fractional) order $s$, and define a "local Sobolev norm" by the formula

$$
\|F\|_{M W_{q}^{s}}=\sup _{t>0}\left\|\eta F_{(t)}\right\|_{W_{q}^{s}}
$$

where $F_{(t)}(\lambda)=F(t \lambda)$, and $\eta \in C_{c}^{\infty}(] 0, \infty[)$ is a not identically zero auxiliary function; note that different choices of $\eta$ give rise to equivalent local norms. Next set $D=$ $\max \left\{d_{1}+d_{2}, 2 d_{2}\right\}$. Then our main result is the following Mihlin-Hörmander-type multiplier theorem.

Theorem 1. Suppose that a function $F: \mathbb{R} \rightarrow \mathbb{C}$ satisfies

$$
\|F\|_{M W_{2}^{s}}<\infty
$$

for some $s>D / 2$. Then the operator $F(L)$ is of weak type $(1,1)$ and bounded on $L^{p}(\mathrm{X})$ for all $\left.p \in\right] 1, \infty[$. In addition,

$$
\|F(L)\|_{L^{1} \rightarrow L^{1, w}} \leq C\|F\|_{M W_{2}^{s}}, \quad\|F(L)\|_{L^{p} \rightarrow L^{p}} \leq C_{p}\|F\|_{M W_{2}^{s}} .
$$

Our approach allows us to consider also the Bochner-Riesz means associated to the Grushin operator, for which we obtain the following result.

Theorem 2. Suppose that $\kappa>(D-1) / 2$ and $p \in[1, \infty]$. Then the Bochner-Riesz means $(1-t L)_{+}^{\kappa}$ are bounded on $L^{p}(\mathrm{X})$ uniformly in $t \in[0, \infty[$.

The main point of interest of Theorems 1 and 2 is that they are sharp, at least if $d_{1} \geq d_{2}$; namely, the lower bounds on the order of differentiability $s$ in Theorem 1 and on the order $\kappa$ of the Bochner-Riesz means in Theorem 2 cannot be decreased. Determining the "critical" orders $s$ and $\kappa$ is commonly regarded as a crucial aspect of the theory of spectral multipliers. For instance, the analogue of Theorem 1 for a sublaplacian on the Heisenberg group has a long history $[4,8,12,21]$ of results requiring a lower and lower order of differentiablity, culminating with the work of Müller and Stein [24] which shows that the critical order is half the topological dimension of the group; see also the independent result by Hebisch [13] for the wider class of Heisenberg-type groups.

The sharpness of Theorems 1 and 2 when $d_{1} \geq d_{2}$ is due to the fact that $D$ in this case coincides with the topological dimension $d_{1}+d_{2}$ of $\mathrm{X}$; since the Grushin operator $L$ is elliptic in the region where $x^{\prime} \neq 0$, one can use the transplantation argument described in [17] to deduce the sharpness of the above theorems from the sharpness of the analogous results for the Laplace operator on $\mathbb{R}^{D}$ (see also [26] for a different argument). In the case $d_{2}>d_{1}$, instead, a gap of $d_{2}-d_{1}$ remains between $D$ and the topological dimension.

If one disregarded the constraints on $s$ and $\kappa$, then the above results would follow from a general theorem $[11,14]$ proved in the context of a doubling metric-measure space $(\mathrm{X}, \varrho, \mu)$, with an operator $L$ satisfying Gaussian-type heat kernel bounds: namely, the weak type $(1,1)$ and $L^{p}$-boundedness for $\left.p \in\right] 1, \infty[$ of $F(L)$ hold whenever $\|F\|_{M W_{\infty}^{s}}<\infty$ for some $s>Q / 2$, where $Q$ denotes the "homogeneous dimension" of the metric-measure space; correspondingly, the uniform $L^{p}$-boundedness for $p \in[1, \infty]$ 
of the Bochner-Riesz means $(1-t L)_{+}^{\kappa}$ holds whenever $\kappa>Q / 2$. As shown in [25], in the case $\mathrm{X}$ is $\mathbb{R}^{d_{1}} \times \mathbb{R}^{d_{2}}$ with Lebesgue measure and $L$ is the Grushin operator, a "control distance" $\varrho$ associated to $L$ can be introduced, and the general theorem applies with $Q=d_{1}+2 d_{2}$.

An alternative approach for the case $d_{2}=1$ is presented in the recent work [16], where the smoothness condition in the Mihlin-Hörmander-type theorem is essentially the same as in [25], while some improvement is obtained for the Bochner-Riesz means (in our notation, their condition is $\kappa>D / 2+1 / 6$ ). However the method used in [16] apparently does not yield the weak type $(1,1)$ in the multiplier theorem, nor the $L^{1}$-boundedness of the Bochner-Riesz means.

Our theorems improve all the mentioned results. In particular, Theorem 1 gives an improvement with respect to both the order of differentiability required on the multiplier (since $D<Q$, even in the case $d_{2}>d_{1}$ ) and the type of Sobolev norm used $\left(L^{2}\right.$ instead of $\left.L^{\infty}\right)$. The use of an $L^{2}$ Sobolev norm is also crucial to obtain $(D-1) / 2$ as the lower bound in Theorem 2 .

When $d_{1}=d_{2}=1$, Theorem 1 proves the conjecture stated on page 5 of [22].

\section{The Grushin operator}

As above, let $\mathrm{X}$ be $\mathbb{R}^{d_{1}} \times \mathbb{R}^{d_{2}}$ with Lebesgue measure. In order to study the Grushin operator $L$ on $\mathrm{X}$, it is convenient to introduce at the same time a family of operators which commute with $L$.

Given a point $x=\left(x^{\prime}, x^{\prime \prime}\right) \in \mathrm{X}$, we denote by $x_{j}^{\prime}$ and $x_{k}^{\prime \prime}$ the $j$ th component of $x^{\prime}$ and the $k$ th component of $x^{\prime \prime}$. For all $j \in\left\{1, \ldots, d_{1}\right\}$ and $k \in\left\{1, \ldots, d_{2}\right\}$, let then $L_{j}$, $T_{k}, P_{j}$ be the differential operators on $\mathrm{X}$ given by

$$
L_{j}=\left(-i \partial_{x_{j}^{\prime}}\right)^{2}+\left(x_{j}^{\prime}\right)^{2} \sum_{l=1}^{d_{2}}\left(-i \partial_{x_{l}^{\prime \prime}}\right)^{2}, \quad T_{k}=-i \partial_{x_{k}^{\prime \prime}}, \quad P_{j}=x_{j}^{\prime} .
$$

If $\left(\delta_{r}\right)_{r>0}$ is the family of dilations on $\mathrm{X}$ defined by

$$
\delta_{r}\left(x^{\prime}, x^{\prime \prime}\right)=\left(r x^{\prime}, r^{2} x^{\prime \prime}\right),
$$

then $\left\|f \circ \delta_{r}\right\|_{2}=r^{-Q / 2}\|f\|_{2}$, where $Q=d_{1}+2 d_{2}$. We also note that

$$
\begin{aligned}
& P_{j}\left(f \circ \delta_{r}\right)=r^{-1}\left(P_{j} f\right) \circ \delta_{r}, \quad L_{j}\left(f \circ \delta_{r}\right)=r^{2}\left(L_{j} f\right) \circ \delta_{r}, \\
& T_{k}\left(f \circ \delta_{r}\right)=r^{2}\left(T_{k} f\right) \circ \delta_{r} .
\end{aligned}
$$

The Grushin operator $L$ on $\mathrm{X}$ is the sum $L_{1}+\cdots+L_{d_{1}} . L$ is a second-order subelliptic differential operator with smooth coefficients. For such operators, several ways of introducing a control distance $\varrho$ are available in the literature, and we refer the reader to [15] for a survey. In particular, $L$ belongs to the class of operators studied in [25], where the following estimates are obtained.

Proposition 3. The control distance $\varrho$ of the Grushin operator L on $\mathrm{X}$ is homogeneous with respect to the dilations $\delta_{r}$, that is,

$$
\varrho\left(\delta_{r}(x), \delta_{r}(y)\right)=r \varrho(x, y)
$$


for all $r>0$ and $x, y \in \mathrm{X}$, and moreover

$$
\varrho(x, y) \sim\left|x^{\prime}-y^{\prime}\right|+ \begin{cases}\frac{\left|x^{\prime \prime}-y^{\prime \prime}\right|}{\left|x^{\prime}\right|+\left|y^{\prime}\right|} & \text { if }\left|x^{\prime \prime}-y^{\prime \prime}\right|^{1 / 2} \leq\left|x^{\prime}\right|+\left|y^{\prime}\right|, \\ \left|x^{\prime \prime}-y^{\prime \prime}\right|^{1 / 2} & \text { if }\left|x^{\prime \prime}-y^{\prime \prime}\right|^{1 / 2} \geq\left|x^{\prime}\right|+\left|y^{\prime}\right| .\end{cases}
$$

Consequently, if $|B(x, r)|$ denotes the Lebesgue measure of the $\varrho$-ball of center $x \in \mathrm{X}$ and radius $r \geq 0$, then

$$
|B(x, r)| \sim r^{d_{1}+d_{2}} \max \left\{r,\left|x^{\prime}\right|\right\}^{d_{2}},
$$

and in particular, for all $\lambda \geq 0$,

$$
|B(x, \lambda r)| \leq C(1+\lambda)^{Q}|B(x, r)| .
$$

Moreover, there exist constants $b, C>0$ such that, for all $t>0$, the integral kernel $p_{t}$ of the operator $\exp (-t L)$ is a function satisfying

$$
\left|p_{t}(x, y)\right| \leq C\left|B\left(y, t^{1 / 2}\right)\right|^{-1} e^{-b \varrho(x, y)^{2} / t}
$$

for all $x, y \in \mathrm{X}$.

Proof. The homogeneity of $\varrho$ follows immediately from its definition [25, Section 4] and the homogeneity of $L$. For the remaining estimates, see [25, Proposition 5.1 and Corollary 6.6].

The inequality (2.4) says that X with the distance $\varrho$ and the Lebesgue measure is a doubling metric-measure space of homogeneous dimension $Q$ (cf. [10, Section 2] or [11, formula (2.2)]), whereas (2.5) expresses Gaussian-type heat kernel bounds for $L$.

\section{The Heisenberg-Reiter group}

Several properties of $L$ and the other operators introduced above can be easily recovered by considering $\mathrm{X}$ as the quotient of a suitable stratified Lie group (cf. $[1,2])$. Denote by $\mathbb{R}^{d_{1} \times d_{2}}$ the set of $\left(d_{1} \times d_{2}\right)$-matrices with real coefficients. Both $\mathbb{R}^{d_{1} \times d_{2}}$ and $\mathbb{R}^{d_{1}} \times \mathbb{R}^{d_{2}}$ are abelian Lie groups with respect to addition. Let $\mathrm{H}_{d_{1}, d_{2}}$ be the semidirect product group $\mathbb{R}^{d_{1} \times d_{2}} \ltimes\left(\mathbb{R}^{d_{1}} \times \mathbb{R}^{d_{2}}\right)$, with multiplication

$$
(x, y, t) \cdot\left(x_{0}, y_{0}, t_{0}\right)=\left(x+x_{0}, y+y_{0}, t+t_{0}-\left(x^{T} y_{0}-x_{0}^{T} y\right) / 2\right) .
$$

This is a particular instance of Heisenberg-Reiter group (see [30] and references therein). If $\left\{\tilde{X}_{1,1}, \ldots, \tilde{X}_{d_{1}, d_{2}}, \tilde{Y}_{1}, \ldots, \tilde{Y}_{d_{1}}, \tilde{T}_{1}, \ldots, \tilde{T}_{d_{2}}\right\}$ is the standard basis of the Lie algebra of $\mathrm{H}_{d_{1}, d_{2}}$ (i.e., the set of the left-invariant vector fields extending the standard basis of $\mathbb{R}^{d_{1} \times d_{2}} \times \mathbb{R}^{d_{1}} \times \mathbb{R}^{d_{2}}$ at the identity), then the only non-trivial Lie brackets among the elements of the basis are

$$
\left[\tilde{X}_{j, k}, \tilde{Y}_{j}\right]=-\left[\tilde{Y}_{j}, \tilde{X}_{j, k}\right]=-\tilde{T}_{k} \quad \text { for all } j=1, \ldots, d_{1}, k=1, \ldots, d_{2} .
$$


$\mathrm{H}_{d_{1}, d_{2}}$ is a 2-step stratified Lie group, with dilations $\left(\tilde{\delta}_{r}\right)_{r>0}$ defined by

$$
\tilde{\delta}_{r}\left(\tilde{X}_{j, k}\right)=r \tilde{X}_{j, k}, \quad \tilde{\delta}_{r}\left(\tilde{Y}_{j}\right)=r \tilde{Y}_{j}, \quad \tilde{\delta}_{r}\left(\tilde{T}_{k}\right)=r^{2} \tilde{T}_{k},
$$

and the homogeneous sublaplacian $\tilde{L}$ on $\mathrm{H}_{d_{1}, d_{2}}$ is given by

$$
\tilde{L}=-\sum_{j, k} \tilde{X}_{j, k}^{2}-\sum_{j} \tilde{Y}_{j}^{2}
$$

Note that, when $d_{2}=1, \mathrm{H}_{d_{1}, d_{2}}$ is the $\left(2 d_{1}+1\right)$-dimensional Heisenberg group. When $d_{2}>1, \mathrm{H}_{d_{1}, d_{2}}$ is not an $\mathrm{H}$-type group (in the sense of Kaplan), nor a Métivier group. Nevertheless, in the terminology of $[18,19], \mathrm{H}_{d_{1}, d_{2}}$ is $h$-capacious where $h=\min \left\{d_{1}, d_{2}\right\}$. In particular, the following multiplier theorem holds: the operator $F(\tilde{L})$ is of weak type $(1,1)$ and bounded on $L^{p}\left(\mathrm{H}_{d_{1}, d_{1}}\right)$ for all $\left.p \in\right] 1, \infty\left[\right.$ whenever $\|F\|_{M W_{2}^{s}}<\infty$ for some $s>\left(\operatorname{dim} \mathrm{H}_{d_{1}, d_{2}}+\left(d_{2}-d_{1}\right)_{+}\right) / 2$, where $\operatorname{dim} \mathrm{H}_{d_{1}, d_{2}}$ is the topological dimension $d_{1} d_{2}+d_{1}+d_{2}[19$, Corollary 6.1].

$\mathrm{X}$ can be identified with the left quotient $\mathbb{R}^{d_{1} \times d_{2}} \backslash \mathrm{H}_{d_{1}, d_{2}}$ via the projection map $(x, y, t) \mapsto\left(y, t+x^{T} y / 2\right)$. Hence, $\mathrm{H}_{d_{1}, d_{2}}$ acts by right translations on $\mathrm{X}$, that is,

$$
\tau_{(x, y, t)}: \mathrm{X} \ni\left(z^{\prime}, z^{\prime \prime}\right) \mapsto\left(z^{\prime}-y, z^{\prime \prime}-x^{T} z^{\prime}-t+x^{T} y / 2\right) \in \mathrm{X}
$$

is a measure-preserving affine transformation of $\mathrm{X}$ for all $(x, y, t) \in \mathrm{H}_{d_{1}, d_{2}}$, and $\tau_{g h}=$ $\tau_{g} \tau_{h}$. This in turn induces a unitary representation $\sigma$ of $\mathrm{H}_{d_{1}, d_{2}}$ on $L^{2}(\mathrm{X})$, given by $\sigma(g) f=f \circ \tau_{g}^{-1}$, and

$$
\begin{array}{r}
T_{k}=d \sigma\left(-i \tilde{T}_{k}\right), \quad P_{j} T_{k}=d \sigma\left(-i \tilde{X}_{j, k}\right), \\
L_{j}=d \sigma\left(-\tilde{Y}_{j}^{2}-\sum_{k} \tilde{X}_{j, k}^{2}\right), \quad L=d \sigma(\tilde{L}) .
\end{array}
$$

This shows in particular that the operators $L_{1}, \ldots, L_{d_{1}}, T_{1}, \ldots, T_{d_{2}}$ (and all the polynomials in $\left.L_{1}, \ldots, L_{d_{1}}, T_{1}, \ldots, T_{d_{2}}\right)$ are essentially self-adjoint on $C_{c}^{\infty}(\mathrm{X})$ and commute strongly (that is, their spectral resolutions commute), so they admit a joint functional calculus on $L^{2}(\mathrm{X})$ in the sense of the spectral theorem [20, Section 3.1]. Arguing analogously, by the use of the unitary representation $\varpi$ of $\mathbb{R}^{d_{1}} \times \mathbb{R}^{d_{2}}$ on $L^{2}(\mathrm{X})$ given by

$$
\left(\varpi\left(u^{\prime}, u^{\prime \prime}\right) f\right)\left(x^{\prime}, x^{\prime \prime}\right)=e^{i\left\langle x^{\prime}, u^{\prime}\right\rangle} f\left(x^{\prime}, x^{\prime \prime}+u^{\prime \prime}\right),
$$

one obtains that the operators $P_{1}, \ldots, P_{d_{1}}, T_{1}, \ldots, T_{d_{2}}$ are essentially self-adjoint on $C_{c}^{\infty}(\mathrm{X})$ and commute strongly.

Because of the mentioned commutation properties, it is convenient to introduce in our notation the following "vectors of operators":

$$
\mathbf{L}=\left(L_{1}, \ldots, L_{d_{1}}\right), \quad \mathbf{T}=\left(T_{1}, \ldots, T_{d_{2}}\right), \quad \mathbf{P}=\left(P_{1}, \ldots, P_{d_{1}}\right) .
$$

Thus, for instance, $|\mathbf{T}|$ stands for the operator $\left(\left|T_{1}\right|^{2}+\cdots+\left|T_{d_{2}}\right|^{2}\right)^{1 / 2}$, that is, the square root $\left(-\Delta_{x^{\prime \prime}}\right)^{1 / 2}$ of minus the second partial Laplacian on $\mathbb{R}^{d_{1}} \times \mathbb{R}^{d_{2}}$, while $|\mathbf{P}|$ is the operator of multiplication by $\left|x^{\prime}\right|$. The subellipticity of $\tilde{L}$ then yields the following estimate. 
Proposition 4. For all $\gamma \in\left[0, \infty\left[\right.\right.$ and $f \in L^{2}(\mathrm{X})$,

$$
\left\||\mathbf{P}|^{\gamma} f\right\|_{2} \leq C_{\gamma}\left\|L^{\gamma / 2}|\mathbf{T}|^{-\gamma} f\right\|_{2},
$$

where the $L^{2}$ norm on each side of (3.2) is understood to be $+\infty$ when $f$ does not belong to the domain of the corresponding operator.

Proof. We may assume $\gamma>0$. Let $\mathbf{P T}$ denote the double-indexed vector of operators $\left(P_{j} T_{k}\right)_{j, k}$, and note that $|\mathbf{P T}|^{\gamma}=|\mathbf{P}|^{\gamma}|\mathbf{T}|^{\gamma}$ (modulo closures). Moreover the spectrum $\left[0,+\infty\left[\right.\right.$ of $|\mathbf{T}|^{\gamma}$ is purely continuous, so $|\mathbf{T}|^{\gamma}$ is injective and its image is dense in $L^{2}(\mathrm{X})$. Therefore (3.2) is reduced to the proof of the inequality

$$
\left\||\mathbf{P T}|^{\gamma} g\right\|_{2} \leq C_{\gamma}\left\|L^{\gamma / 2} g\right\|_{2}
$$

for all $g \in L^{2}(\mathrm{X})$.

By (3.1), the differential operator $\tilde{W}=-\sum_{j, k} \tilde{X}_{j, k}^{2}$ on $\mathrm{H}_{d_{1}, d_{2}}$ corresponds to the operator $|\mathbf{P T}|^{2}$ on $\mathrm{X}$. Since $\tilde{W}$ is $\tilde{\delta}_{r}$-homogeneous, with the same homogeneity degree as the sublaplacian $\tilde{L}$, from (3.1) and [28, Theorem 2.5] we deduce (3.3) for all $\gamma \in$ $2 \mathbb{N}$ and $g \in L^{2}(\mathrm{X})$. The extension of (3.3) to all the real $\gamma \geq 0$ is an immediate consequence of the Löwner-Heinz inequality (see, e.g., [5, Section I.5] and references therein).

\section{Weighted Plancherel estimates}

As in many other works on the subject, the proof of our results is based on the analysis of the integral kernel $\mathcal{K}_{F(L)}: \mathrm{X} \times \mathrm{X} \rightarrow \mathbb{C}$ of the operator $F(L)$, defined by the identity

$$
F(L) f(x)=\int_{\mathrm{X}} \mathcal{K}_{F(L)}(x, y) f(y) d y .
$$

To be precise, if $F$ is bounded and compactly supported, then there exists a Borel function $\mathcal{K}_{F(L)}$ such that (4.1) holds for all $f \in L^{2}(\mathrm{X})$ and for almost all $x \in \mathrm{X}$ (cf. [11, Lemma 2.2]). However, a multiplier $F$ satisfying (1.1) need not be compactly supported, and the integral kernel $\mathcal{K}_{F(L)}$ in general exists only as a distribution; nevertheless the Calderón-Zygmund theory of singular integral operators allows one to derive the weak type $(1,1)$ of $F(L)$ from suitable estimates on the integral kernels corresponding to the compactly supported pieces in a dyadic decomposition of $F$.

As highlighted in [11], a crucial step in this approach is a "Plancherel estimate," which in its basic form is the inequality

$$
\underset{y \in \mathrm{X}}{\operatorname{ess} \sup }\left|B\left(y, R^{-1}\right)\right|^{1 / 2}\left\|\mathcal{K}_{F(L)}(\cdot, y)\right\|_{L^{2}(\mathrm{X})} \leq C\left\|F_{\left(R^{2}\right)}\right\|_{L^{\infty}(\mathbb{R})},
$$

for all $R>0$ and all $F: \mathbb{R} \rightarrow \mathbb{C}$ supported in the interval $\left[R^{2}, 4 R^{2}\right]$. Such an estimate holds, mutatis mutandis, for any operator $L$ satisfying Gaussian heat kernel bounds, but usually it does not lead to optimal spectral multiplier results. In the present paper we obtain for the Grushin operator $L$ an improvement of (4.2), that is, a "weighted 
Plancherel estimate" of the form

$$
\underset{y \in \mathrm{X}}{\operatorname{ess} \sup }\left|B\left(y, R^{-1}\right)\right|^{1 / 2}\left\|\left(1+w_{R}(\cdot, y)\right)^{\gamma} \mathcal{K}_{F(L)}(\cdot, y)\right\|_{L^{2}(\mathrm{X})} \leq C_{\gamma}\left\|F_{\left(R^{2}\right)}\right\|_{L^{2}(\mathbb{R})},
$$

where $\gamma \in\left[0, d_{2} / 2[\right.$ and

$$
w_{R}(x, y)=\min \left\{R,\left|y^{\prime}\right|^{-1}\right\}\left|x^{\prime}\right| .
$$

The improvement of the Plancherel estimate yields, via the interpolation technique of [21], a sharp multiplier theorem, at least for $d_{1} \leq d_{2}$. In the case $d_{1}>d_{2}$, an interesting phenomenon occurs: although (4.3) holds for all $\gamma \in\left[0, d_{2} / 2\right.$ [, we can exploit it only when $\gamma<d_{1} / 2$; whence the gap between the threshold $D / 2$ in Theorem 1 and half the topological dimension. The rest of this section is devoted to the derivation of (4.3).

From the previous section we know that the operators $L_{1}, \ldots, L_{d_{1}}, T_{1}, \ldots, T_{d_{2}}$ have a joint functional calculus. In fact one can obtain a quite explicit formula for the integral kernel $\mathcal{K}_{G(\mathbf{L}, \mathbf{T})}$ of an operator $G(\mathbf{L}, \mathbf{T})$ in the functional calculus, in terms of the Hermite functions (cf. [22, Proposition 3.1] for the case $d_{1}=d_{2}=1$, and [27] for the analogue on the Heisenberg groups). Namely, for all $\ell \in \mathbb{N}$, let $h_{\ell}$ denote the $\ell$ th Hermite function, that is,

$$
h_{\ell}(t)=(-1)^{\ell}\left(\ell ! 2^{\ell} \sqrt{\pi}\right)^{-1 / 2} e^{t^{2} / 2}\left(\frac{d}{d t}\right)^{\ell} e^{-t^{2}}
$$

and set, for all $n \in \mathbb{N}^{d_{1}}, u \in \mathbb{R}^{d_{1}}, \xi \in \mathbb{R}^{d_{2}}$,

$$
\tilde{h}_{n}(u, \xi)=|\xi|^{d_{1} / 4} h_{n_{1}}\left(|\xi|^{1 / 2} u_{1}\right) \cdots h_{n_{d_{1}}}\left(|\xi|^{1 / 2} u_{d_{1}}\right) .
$$

Proposition 5. For all bounded Borel functions $G: \mathbb{R}^{d_{1}} \times \mathbb{R}^{d_{2}} \rightarrow \mathbb{C}$ compactly supported in $\mathbb{R}^{d_{1}} \times\left(\mathbb{R}^{d_{2}} \backslash\{0\}\right)$,

$$
\mathcal{K}_{G(\mathbf{L}, \mathbf{T})}(x, y)=(2 \pi)^{-d_{2}} \int_{\mathbb{R}^{d_{2}}} \sum_{n \in \mathbb{N}^{d_{1}}} G(|\xi|(2 n+\tilde{1}), \xi) \tilde{h}_{n}\left(y^{\prime}, \xi\right) \tilde{h}_{n}\left(x^{\prime}, \xi\right) e^{i\left\langle\xi, x^{\prime \prime}-y^{\prime \prime}\right\rangle} d \xi
$$

for almost all $x, y \in \mathrm{X}$, where $\tilde{1}=(1, \ldots, 1) \in \mathbb{N}^{d_{1}}$. In particular

$$
\left\|\mathcal{K}_{G(\mathbf{L}, \mathbf{T})}(\cdot, y)\right\|_{2}^{2}=(2 \pi)^{-d_{2}} \int_{\mathbb{R}^{d_{2}}} \sum_{n \in \mathbb{N}^{d_{1}}}|G(|\xi|(2 n+\tilde{1}), \xi)|^{2} \tilde{h}_{n}^{2}\left(y^{\prime}, \xi\right) d \xi
$$

for almost all $y \in \mathrm{X}$.

Proof. Let $\mathcal{F}: L^{2}\left(\mathbb{R}^{d_{1}} \times \mathbb{R}^{d_{2}}\right) \rightarrow L^{2}\left(\mathbb{R}^{d_{1}} \times \mathbb{R}^{d_{2}}\right)$ be the isometry defined by

$$
\mathcal{F} \phi\left(x^{\prime}, \xi\right)=(2 \pi)^{-d_{2} / 2} \int_{\mathbb{R}^{d_{2}}} \phi\left(x^{\prime}, x^{\prime \prime}\right) e^{-i\left\langle\xi, x^{\prime \prime}\right\rangle} d x^{\prime \prime},
$$

i.e., the Fourier transform with respect to $x^{\prime \prime}$. Then

$$
\mathcal{F} L_{j} \phi\left(x^{\prime}, \xi\right)=L_{j, \xi} \mathcal{F} \phi\left(x^{\prime}, \xi\right), \quad \mathcal{F} T_{k} \phi\left(x^{\prime}, \xi\right)=\xi_{k} \mathcal{F} \phi\left(x^{\prime}, \xi\right),
$$


at least for $\phi$ in the Schwartz class, where

$$
L_{j, \xi}=\left(-i \partial_{x_{j}^{\prime}}\right)^{2}+|\xi|^{2}\left(x_{j}^{\prime}\right)^{2}
$$

For all $\xi \neq 0,\left\{\tilde{h}_{n}(\cdot, \xi)\right\}_{n \in \mathbb{N}^{d_{1}}}$ is a complete orthonormal system for $L^{2}\left(\mathbb{R}^{d_{1}}\right)$ made of real-valued functions and

$$
L_{j, \xi} \tilde{h}_{n}\left(x^{\prime}, \xi\right)=\left(2 n_{j}+1\right)|\xi| \tilde{h}_{n}\left(x^{\prime}, \xi\right) .
$$

In particular, if $\mathcal{G}: L^{2}\left(\mathbb{R}^{d_{1}} \times \mathbb{R}^{d_{2}}\right) \rightarrow L^{2}\left(\mathbb{N}^{d_{1}} \times \mathbb{R}^{d_{2}}\right)$ is the isometry defined by

$$
\mathcal{G} \psi(n, \xi)=\int_{\mathbb{R}^{d_{1}}} \psi\left(x^{\prime}, \xi\right) \tilde{h}_{n}\left(x^{\prime}, \xi\right) d x^{\prime},
$$

then

$$
\mathcal{G F} L_{j} \phi(n, \xi)=\left(2 n_{j}+1\right)|\xi| \mathcal{G F} \phi(n, \xi), \quad \mathcal{G} \mathcal{F} T_{k} \phi(n, \xi)=\xi_{k} \mathcal{G F} \phi(n, \xi) .
$$

The isometry $\mathcal{G} \mathcal{F}$ intertwines the operators $L_{j}$ and $T_{k}$ with some multiplication operators on $\mathbb{N}^{d_{1}} \times \mathbb{R}^{d_{2}}$, hence it intertwines the corresponding functional calculi:

$$
\mathcal{G F} G(\mathbf{L}, \mathbf{T}) \phi(n, \xi)=G(|\xi|(2 n+\tilde{1}), \xi) \mathcal{G} \mathcal{F} \phi(n, \xi) .
$$

The inversion formulae for $\mathcal{F}$ and $\mathcal{G}$ and some easy manipulations then give the above expression for $\mathcal{K}_{G(\mathbf{L}, \mathbf{T})}$. Moreover, if we set

$$
G_{y}(n, \xi)=(2 \pi)^{-d_{2} / 2} G(|\xi|(2 n+\tilde{1}), \xi) \tilde{h}_{n}\left(y^{\prime}, \xi\right) e^{-i\left\langle\xi, y^{\prime \prime}\right\rangle},
$$

then the formula for $\mathcal{K}_{G(\mathbf{L}, \mathbf{T})}$ can be rewritten as

$$
\mathcal{K}_{G(\mathbf{L}, \mathbf{T})}(\cdot, y)=(\mathcal{G F})^{-1} G_{y},
$$

and since $\mathcal{G F}: L^{2}(\mathrm{X}) \rightarrow L^{2}\left(\mathbb{N}^{d_{1}} \times \mathbb{R}^{d_{2}}\right)$ is an isometry we obtain (4.5).

If we restrict to the joint functional calculus of $L, T_{1}, \ldots, T_{d_{2}}$, the formula (4.5) can be rewritten as follows. For all positive integers $d$, set $\mathbb{N}_{d}=2 \mathbb{N}+d$, and define, for all $N \in \mathbb{N}_{d}$ and $u \in \mathbb{R}^{d}$,

$$
H_{d, N}(u)=\sum_{\substack{n_{1}, \ldots, n_{d} \in \mathbb{N} \\ 2 n_{1}+\cdots+2 n_{d}+d=N}} h_{n_{1}}^{2}\left(u_{1}\right) \cdots h_{n_{d}}^{2}\left(u_{d}\right) .
$$

Corollary 6. For all bounded Borel functions $G: \mathbb{R} \times \mathbb{R}^{d_{2}} \rightarrow \mathbb{C}$ compactly supported in $\mathbb{R} \times\left(\mathbb{R}^{d_{2}} \backslash\{0\}\right)$,

$$
\left\|\mathcal{K}_{G(L, \mathbf{T})}(\cdot, y)\right\|_{2}^{2}=(2 \pi)^{-d_{2}} \int_{\mathbb{R}^{d_{2}}} \sum_{N \in \mathbb{N}_{d_{1}}}|G(N|\xi|, \xi)|^{2}|\xi|^{d_{1} / 2} H_{d_{1}, N}\left(|\xi|^{1 / 2} y^{\prime}\right) d \xi
$$

for almost all $y \in \mathrm{X}$.

We can now combine (3.2) and (4.6) to get the following weighted inequalities. 
Proposition 7. For all $\gamma \geq 0$ and for all compactly supported bounded Borel functions $F: \mathbb{R} \rightarrow \mathbb{C}$,

$$
\left\||\mathbf{P}|^{\gamma} \mathcal{K}_{F(L)}(\cdot, y)\right\|_{2}^{2} \leq C_{\gamma} \int_{0}^{\infty}|F(\lambda)|^{2} \sum_{N \in \mathbb{N}_{d_{1}}} \frac{\lambda^{Q / 2-\gamma}}{N^{Q / 2-2 \gamma}} H_{d_{1}, N}\left(\frac{\lambda^{1 / 2} y^{\prime}}{N^{1 / 2}}\right) \frac{d \lambda}{\lambda}
$$

for almost all $y \in \mathrm{X}$.

Proof. Let $G: \mathbb{R} \times \mathbb{R}^{d_{2}} \rightarrow \mathbb{C}$ be as in Corollary 6 . In particular $\mathcal{K}_{G(L, \mathbf{T})}(\cdot, y) \in L^{2}(\mathrm{X})$ for almost all $y \in \mathrm{X}$, and from [9, Theorem III.6.20] and the definition of integral kernel one may deduce

$$
L^{\gamma / 2}|\mathbf{T}|^{-\gamma}\left(\mathcal{K}_{G(L, \mathbf{T})}(\cdot, y)\right)=\mathcal{K}_{L^{\gamma / 2}|\mathbf{T}|^{-\gamma} G(L, \mathbf{T})}(\cdot, y)
$$

for all $\gamma \geq 0$ and almost all $y \in \mathrm{X}$. This equality, together with (4.6) and (3.2), implies that

$$
\left\||\mathbf{P}|^{\gamma} \mathcal{K}_{G(L, \mathbf{T})}(\cdot, y)\right\|_{2}^{2} \leq C_{\gamma} \sum_{N \in \mathbb{N}_{d_{1}}} \int_{\mathbb{R}^{d_{2}}}|G(N|\xi|, \xi)|^{2} N^{\gamma}|\xi|^{d_{1} / 2-\gamma} H_{d_{1}, N}\left(|\xi|^{1 / 2} y^{\prime}\right) d \xi
$$

Choose now an increasing sequence $\left(\zeta_{n}\right)_{n \in \mathbb{N}}$ of non-negative Borel functions on $\mathbb{R}$, compactly supported in $\mathbb{R} \backslash\{0\}$ and converging pointwise on $\mathbb{R} \backslash\{0\}$ to the constant 1 , and define $G_{n}(\lambda, \xi)=F(\lambda) \zeta_{n}(|\xi|)$. Note that $\mathcal{K}_{F(L)}(\cdot, y) \in L^{2}(\mathrm{X})$ for almost all $y \in \mathrm{X}[11$, Lemma 2.2], hence

$$
\mathcal{K}_{G_{n}(L, \mathbf{T})}(\cdot, y)=\zeta_{n}(|\mathbf{T}|)\left(\mathcal{K}_{F(L)}(\cdot, y)\right)
$$

for almost all $y \in \mathrm{X}$, as before, and $\mathcal{K}_{G_{n}(L, \mathbf{T})}(\cdot, y) \rightarrow \mathcal{K}_{F(L)}(\cdot, y)$ in $L^{2}(\mathrm{X})$ for almost all $y$, because $|\mathbf{T}|$ has trivial kernel. The conclusion then follows by applying the previous inequality when $G=G_{n}$ and letting $n$ tend to infinity.

Now we recall some well-known estimates for the Hermite functions, which we need in the sequel.

Lemma 8. For all $N=2 n+1 \in \mathbb{N}_{1}$,

$$
H_{1, N}(u)=h_{n}^{2}(u) \leq \begin{cases}C\left(N^{1 / 3}+\left|u^{2}-N\right|\right)^{-1 / 2} & \text { for all } u \in \mathbb{R}, \\ C \exp \left(-c u^{2}\right) & \text { when } u^{2} \geq 2 N .\end{cases}
$$

Moreover, if $d \geq 2$, then, for all $N \in \mathbb{N}_{d}$,

$$
H_{d, N}(u) \leq \begin{cases}C N^{d / 2-1} & \text { for all } u \in \mathbb{R}^{d}, \\ C \exp \left(-c|u|_{\infty}^{2}\right) & \text { when }|u|_{\infty}^{2} \geq 2 N\end{cases}
$$

where $|u|_{\infty}=\max \left\{\left|u_{1}\right|, \ldots,\left|u_{d}\right|\right\}$.

Proof. For the bounds (4.8), see [23, (2.3), p. 435] or [29, Lemma 1.5.1]. For the first inequality in (4.9), see [29, Lemma 3.2.2]; the second inequality is an easy consequence of (4.8).

These bounds allow us to obtain the following crucial estimate. 
Lemma 9. For all fixed $d \in \mathbb{N} \backslash\{0\}$ and $\varepsilon \in] 0, \infty[$, the sum

$$
\sum_{N \in \mathbb{N}_{d}} \frac{\max \{1,|u|\}^{\varepsilon}}{N^{d / 2+\varepsilon}} H_{d, N}\left(\frac{u}{N^{1 / 2}}\right)
$$

has a finite upper bound, independent of $u \in \mathbb{R}^{d}$.

Proof. We split the sum into several parts, and use the bounds (4.8), (4.9).

The part where $N \leq|u| / 2$ is empty unless $|u| \geq 1$; in this case, moreover, $\left|N^{-1 / 2} u\right|^{2} \geq 4 N$, hence $H_{d, N}\left(N^{-1 / 2} u\right) \leq C \exp \left(-c|u|^{2} / N\right)$, and

$$
\sup _{u} \sum_{N \leq|u| / 2}|u|^{\varepsilon} N^{-d / 2-\varepsilon} \exp \left(-c|u|^{2} / N\right) \leq \sum_{N \in \mathbb{N}_{d}} \sup _{t \geq 4 N} t^{\varepsilon / 2} \exp (-c t),
$$

which is finite.

If $N \geq|u| / 2$ and $d \geq 2$, then $H_{d, N}\left(N^{-1 / 2} u\right) \leq C N^{d / 2-1}$, and

$$
\sup _{u} \sum_{N \geq|u| / 2} \max \{1,|u|\}^{\varepsilon} N^{-1-\varepsilon}<\infty .
$$

When $d=1$, the same argument works for the part where $N \geq 2|u|$, because in this case $\left|N^{-1 / 2} u\right|^{2} \leq N / 4$ and the bound $H_{N}\left(N^{-1 / 2} u\right) \leq C N^{-1 / 2}$ holds. However the part where $|u| / 2<N<2|u|$ requires a different estimate.

Namely, the part of (4.10) where $|u| / 2<N \leq|u|-1$ is majorized by

$$
\left.C_{\varepsilon}|u|^{-1} \sum_{|u| / 2<N \leq|u|-1}|1-N /| u\right|^{-1 / 2} \leq C_{\varepsilon} \int_{1 / 2}^{1}|1-t|^{-1 / 2} d t,
$$

which is finite and independent of $u$. Analogously one bounds the part of (4.10) where $|u|+1 \leq N<2|u|$. The remaining part, where $|u|-1<N<|u|+1$, contains at most one summand, which moreover is bounded by a constant.

The previous inequality allows us to simplify (4.7) considerably and to obtain the announced weighted Plancherel estimates. Recall that $w_{R}$ denotes the weight function defined by (4.4) for all $R>0$.

Proposition 10. For all $\gamma \in\left[0, d_{2} / 2[\right.$ and all bounded compactly supported Borel functions $F: \mathbb{R} \rightarrow \mathbb{C}$,

$$
\left\||\mathbf{P}|^{\gamma} \mathcal{K}_{F(L)}(\cdot, y)\right\|_{2}^{2} \leq C_{\gamma} \int_{0}^{\infty}|F(\lambda)|^{2} \lambda^{\left(d_{1}+d_{2}\right) / 2} \min \left\{\lambda^{d_{2} / 2-\gamma},\left|y^{\prime}\right|^{2 \gamma-d_{2}}\right\} \frac{d \lambda}{\lambda}
$$

for almost all $y \in \mathrm{X}$. In particular, for all $R>0$, if $\operatorname{supp} F \subseteq\left[R^{2}, 4 R^{2}\right]$, then

$$
\underset{y \in \mathrm{X}}{\operatorname{ess} \sup }\left|B\left(y, R^{-1}\right)\right|^{1 / 2}\left\|\left(1+w_{R}(\cdot, y)\right)^{\gamma} \mathcal{K}_{F(L)}(\cdot, y)\right\|_{2} \leq C_{\gamma}\left\|F_{\left(R^{2}\right)}\right\|_{L^{2}},
$$

where the constant $C_{\gamma}$ does not depend on $R$.

Proof. In view of (4.7), the first inequality follows immediately from Lemma 9 with $d=d_{1}$ and $\varepsilon=d_{2}-2 \gamma$. In the case $\operatorname{supp} F \subseteq\left[R^{2}, 4 R^{2}\right]$, a simple manipulation, together with (4.4) and (2.3), gives the second inequality. 


\section{The multiplier theorems}

We now show how the weighted Plancherel estimates obtained in the previous section can be used to improve the known multiplier theorems for the Grushin operator. First we recall the basic known estimates for operators satisfying Gaussian-type heat kernel bounds in a doubling metric-measure space.

Proposition 11. For all $R>0, \alpha \geq 0, \beta>\alpha$, and for all functions $F: \mathbb{R} \rightarrow \mathbb{C}$ such that $\operatorname{supp} F \subseteq\left[-4 R^{2}, 4 R^{2}\right]$,

$$
\underset{y \in \mathrm{X}}{\operatorname{ess} \sup }\left|B\left(y, R^{-1}\right)\right|^{1 / 2}\left\|(1+R \varrho(\cdot, y))^{\alpha} \mathcal{K}_{F(L)}(\cdot, y)\right\|_{2} \leq C_{\alpha, \beta}\left\|F_{\left(R^{2}\right)}\right\|_{W_{\infty}^{\beta}},
$$

where the constant $C_{\alpha, \beta}$ does not depend on $R$. If in addition $\beta>\alpha+Q / 2$, then

$$
\underset{y \in \mathrm{X}}{\operatorname{ess} \sup }\left\|(1+R \varrho(\cdot, y))^{\alpha} \mathcal{K}_{F(L)}(\cdot, y)\right\|_{1} \leq C_{\alpha, \beta}\left\|F_{\left(R^{2}\right)}\right\|_{W_{\infty}^{\beta}},
$$

where again $C_{\alpha, \beta}$ does not depend on $R$.

Proof. For the first inequality, see [14] or [11, Lemma 4.3]; note that the statement in [11] seems to require that the multiplier $F$ is supported away from the origin, but its proof clarifies that this is not necessary, because here we do not perform the change of variable $\lambda \mapsto \sqrt{\lambda}$ in the multiplier function. The second inequality is an immediate consequence of the first, via Hölder's inequality and [11, Lemma 4.4].

These inequalities can be improved by means of the weighted Plancherel estimates. For this, some properties of the weight functions $w_{R}$ are needed.

Lemma 12. Suppose that $0 \leq \gamma<\min \left\{d_{1}, d_{2}\right\} / 2$ and $\beta>Q / 2-\gamma$. For all $y \in \mathrm{X}$ and $R>0$,

$$
\int_{\mathrm{X}}\left(1+w_{R}(x, y)\right)^{-2 \gamma}(1+R \varrho(x, y))^{-2 \beta} d x \leq C_{\gamma, \beta}\left|B\left(y, R^{-1}\right)\right| .
$$

Moreover, for all $x, y \in \mathrm{X}$ and $R>0$,

$$
w_{R}(x, y) \leq C(1+R \varrho(x, y)) .
$$

Proof. By exploiting the homogeneity properties of the distance $\varrho$ and the weights $w_{R}$, we may suppose that $R=1$. Then (5.4) immediately follows from the fact that

$$
\min \left\{1,\left|y^{\prime}\right|^{-1}\right\}\left|x^{\prime}\right| \leq 1+\left|x^{\prime}-y^{\prime}\right| \leq C(1+\varrho(x, y)),
$$

by $(2.2)$.

To show (5.3) we note that by translation-invariance we may also suppose that $y^{\prime \prime}=0$. By $(2.3)$, we must then prove that

$$
\int_{\mathrm{X}}\left(1+\frac{\left|x^{\prime}-y^{\prime}\right|}{1+\left|y^{\prime}\right|}\right)^{-2 \gamma}(1+\varrho(x, y))^{-2 \beta} d x \leq C_{\gamma, \beta}\left(1+\left|y^{\prime}\right|\right)^{d_{2}} .
$$


We split the integral into two parts, according to the asymptotics (2.2). In the region $\mathrm{X}_{1}=\left\{x \in \mathrm{X}:\left|x^{\prime \prime}\right|^{1 / 2} \geq\left|x^{\prime}\right|+\left|y^{\prime}\right|\right\}$, we decompose $\beta=\beta_{1}+\beta_{2}$ so that $\beta_{1}>d_{1} / 2-\gamma$ and $\beta_{2}>d_{2}$, whence the integral on $\mathrm{X}_{1}$ is at most

$$
C_{\beta}\left(1+\left|y^{\prime}\right|\right)^{2 \gamma} \int_{\mathbb{R}^{d_{1}}}\left(1+\left|x^{\prime}-y^{\prime}\right|\right)^{-2\left(\gamma+\beta_{1}\right)} d x^{\prime} \int_{\mathbb{R}^{d_{2}}}\left(1+\left|x^{\prime \prime}\right|^{1 / 2}\right)^{-2 \beta_{2}} d x^{\prime \prime} .
$$

In the region $\mathrm{X}_{2}=\left\{x \in \mathrm{X}:\left|x^{\prime \prime}\right|^{1 / 2}<\left|x^{\prime}\right|+\left|y^{\prime}\right|\right\}$, instead, we decompose $\beta=\tilde{\beta}_{1}+\tilde{\beta}_{2}$ so that $\tilde{\beta}_{1}>\left(d_{1}+d_{2}\right) / 2-\gamma$ and $\tilde{\beta}_{2}>d_{2} / 2$, whence the integral on $\mathrm{X}_{2}$ is at most

$$
\begin{aligned}
C_{\beta} \int_{\mathrm{X}} & \left(1+\frac{\left|x^{\prime}-y^{\prime}\right|}{1+\left|y^{\prime}\right|}\right)^{-2 \gamma}\left(1+\left|x^{\prime}-y^{\prime}\right|\right)^{-2 \tilde{\beta}_{1}}\left(1+\frac{\left|x^{\prime \prime}\right|}{\left|x^{\prime}\right|+\left|y^{\prime}\right|}\right)^{-2 \tilde{\beta}_{2}} d x \\
& \leq C_{\gamma, \beta} \int_{\mathbb{R}^{d_{1}}}\left(1+\frac{|u|}{1+\left|y^{\prime}\right|}\right)^{-2 \gamma}(1+|u|)^{-2 \tilde{\beta}_{1}}\left(\left|u+y^{\prime}\right|+\left|y^{\prime}\right|\right)^{d_{2}} d u \\
& \leq C_{\gamma, \beta}\left(\left(1+\left|y^{\prime}\right|\right)^{2 \gamma} \int_{\mathbb{R}^{d_{1}}}(1+|u|)^{-2 \nu} d u+\left|y^{\prime}\right|^{d_{2}} \int_{\mathbb{R}^{d_{1}}}(1+|u|)^{-2 \tilde{\beta}_{1}} d u\right),
\end{aligned}
$$

where $\nu=\tilde{\beta}_{1}+\gamma-d_{2} / 2>d_{1} / 2$. The conclusion follows.

A strengthened weighted version of (5.1) can now be obtained using the MauceriMeda interpolation trick [21] (see also [19, Section 3] and [11, Lemma 4.3]).

Proposition 13. For all $R>0, \alpha \geq 0, \beta>\alpha, \gamma \in\left[0, d_{2} / 2[\right.$, and for all functions $F: \mathbb{R} \rightarrow \mathbb{C}$ such that $\operatorname{supp} F \subseteq\left[R^{2}, 4 R^{2}\right]$,

$$
\begin{aligned}
& \underset{y \in \mathrm{X}}{\operatorname{ess} \sup }\left|B\left(y, R^{-1}\right)\right|^{1 / 2}\left\|(1+R \varrho(\cdot, y))^{\alpha}\left(1+w_{R}(\cdot, y)\right)^{\gamma} \mathcal{K}_{F(L)}(\cdot, y)\right\|_{2} \\
& \quad \leq C_{\alpha, \beta, \gamma}\left\|F_{\left(R^{2}\right)}\right\|_{W_{2}^{\beta}},
\end{aligned}
$$

where the constant $C_{\alpha, \beta, \gamma}$ does not depend on $R$.

Proof. The estimate (5.1), together with (5.4) and a Sobolev embedding, immediately implies (5.5) in the case $\beta>\alpha+d_{2} / 2+1 / 2$. On the other hand, in the case $\alpha=0,(5.5)$ is given by Proposition 10 for all $\beta>0$. The conclusion then follows by interpolation (see, e.g., $[3,7]$ ).

An alternative proof of Proposition 13 can be obtained using minor adjustments of the technique developed in [6].

Let $D=Q-\min \left\{d_{1}, d_{2}\right\}=\max \left\{d_{1}+d_{2}, 2 d_{2}\right\}$. Proposition 13, together with (5.3) and Hölder's inequality, then yields an improvement of (5.2).

Corollary 14. For all $R>0, \alpha \geq 0, \beta>\alpha+D / 2$, and for all functions $F: \mathbb{R} \rightarrow \mathbb{C}$ such that $\operatorname{supp} F \subseteq\left[R^{2}, 4 R^{2}\right]$,

$$
\underset{y \in \mathrm{X}}{\operatorname{ess} \sup }\left\|(1+R \varrho(\cdot, y))^{\alpha} \mathcal{K}_{F(L)}(\cdot, y)\right\|_{1} \leq C_{\alpha, \beta}\left\|F_{\left(R^{2}\right)}\right\|_{W_{2}^{\beta}},
$$

where the constant $C_{\alpha, \beta}$ does not depend on $R$. In particular, under the same hypotheses,

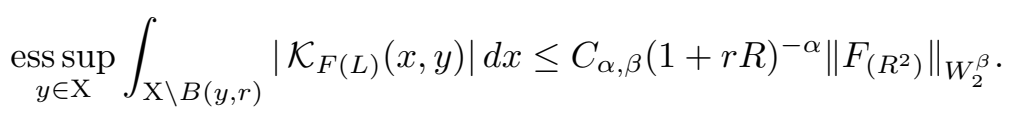


We are finally able to prove our main results.

Proof of Theorem 1. We can follow the lines of the proof of [11, Theorem 3.1], where the inequality (4.18) there is replaced by our (5.7).

Proof of Theorem 2. Choose $\beta \in] D / 2, \kappa+1 / 2\left[\right.$. Let $\eta \in C_{c}^{\infty}(\mathbb{R})$ be supported in $[-1 / 2,1 / 2]$ and equal to 1 in a neighborhood of the origin, and set $F(\lambda)=(1-|\lambda|)_{+}^{\kappa}$. The function $\eta F$ is smooth and compactly supported, while $(1-\eta) F$ is compactly supported away from the origin and belongs to $W_{2}^{\beta}$. The inequalities (5.2) and (5.6) then imply that the operators $\eta(t L) F(t L)$ and $(1-\eta(t L)) F(t L)$ are bounded on $L^{1}(\mathrm{X})$, uniformly in $t>0$, and the same holds for their sum $(1-t L)_{+}^{\kappa}$. The conclusion follows by self-adjointness and interpolation.

\section{Acknowledgments}

This research was supported by Australian Research Council Discovery grants DP110102488 (A.M., A.S.). The first-named author thanks the Australian Research Council and the Alexander von Humboldt Foundation for support when this work was begun and when it was finished, and the University of New South Wales and the Christian-Albrechts-Universität zu Kiel for their hospitality.

\section{References}

[1] N. Arcozzi and A. Baldi, From Grushin to Heisenberg via an isoperimetric problem, J. Math. Anal. Appl. 340(1) (2008), 165-174.

[2] R. Beals, B. Gaveau, P. Greiner and Y. Kannai, Transversally elliptic operators, Bull. Sci. Math. 128(7) (2004), 531-576.

[3] J. Bergh and J. Löfström, Interpolation spaces, An introduction, Springer-Verlag, Berlin (1976). Grundlehren der Mathematischen Wissenschaften, No. 223.

[4] M. Christ, $L^{p}$ bounds for spectral multipliers on nilpotent groups, Trans. Amer. Math. Soc. 328(1) (1991), 73-81.

[5] H.O. Cordes, Spectral theory of linear differential operators and comparison algebras, Vol. 76 of London Mathematical Society Lecture Note Series, Cambridge University Press, Cambridge (1987).

[6] M. Cowling and A. Sikora, A spectral multiplier theorem for a sublaplacian on SU(2), Math. Z. 238(1) (2001), 1-36.

[7] M. Cwikel and S. Janson, Interpolation of analytic families of operators, Studia Math. 79(1) (1984), 61-71.

[8] L. De Michele and G. Mauceri, $L^{p}$ multipliers on the Heisenberg group, Michigan Math. J. 26(3) (1979), 361-371.

[9] N. Dunford and J.T. Schwartz, Linear Operators. I. General Theory, Vol. 7 of Pure and Applied Mathematics, Interscience Publishers, Inc., New York (1958).

[10] X.T. Duong and A. McIntosh, Singular integral operators with non-smooth kernels on irregular domains, Rev. Mat. Iberoamericana 15(2) (1999), 233-265.

[11] X.T. Duong, E.M. Ouhabaz and A. Sikora, Plancherel-type estimates and sharp spectral multipliers, J. Funct. Anal. 196(2) (2002), 443-485.

[12] G.B. Folland and E.M. Stein, Hardy spaces on homogeneous groups, Vol. 28 of Mathematical Notes, Princeton University Press, Princeton, N.J. (1982).

[13] W. Hebisch, Multiplier theorem on generalized Heisenberg groups, Colloq. Math. 65(2) (1993), 231-239.

[14] - Functional calculus for slowly decaying kernels (1995). Preprint. Available on the web at http://www.math.uni.wroc.pl/ hebisch/. 
[15] D. Jerison and A. Sánchez-Calle, Subelliptic, second order differential operators, in Complex analysis, III (College Park, Md., 1985-86), Vol. 1277 of Lecture Notes in Math., 46-77, Springer, Berlin (1987).

[16] K. Jotsaroop, P.K. Sanjay and S. Thangavelu, Riesz transforms and multipliers for the Grushin operator (2011). To appear in J. Anal. Math. arXiv:1110.3227.

[17] C.E. Kenig, R.J. Stanton and P.A. Tomas, Divergence of eigenfunction expansions, J. Funct. Anal. 46(1) (1982), 28-44.

[18] A. Martini, Algebras of differential operators on Lie groups and spectral multipliers, Tesi di perfezionamento (PhD thesis), Scuola Normale Superiore, Pisa (2010). arXiv:1007.1119.

[19] - Analysis of joint spectral multipliers on Lie groups of polynomial growth, Ann. Inst. Fourier (Grenoble) 62(4) (2012), 1215-1263.

[20] - Spectral theory for commutative algebras of differential operators on Lie groups, J. Funct. Anal. 260(9) (2011), 2767-2814.

[21] G. Mauceri and S. Meda, Vector-valued multipliers on stratified groups, Rev. Mat. Iberoamericana 6(3-4) (1990), 141-154.

[22] R. Meyer, $L^{p}$-estimates for the wave equation associated to the Grušin operator, PhD dissertation, Christian-Albrechts-Universität zu Kiel (2006).

[23] B. Muckenhoupt, Mean convergence of Hermite and Laguerre series. II, Trans. Amer. Math. Soc. 147 (1970), 433-460.

[24] D. Müller and E.M. Stein, On spectral multipliers for Heisenberg and related groups, J. Math. Pures Appl. (9) 73(4) (1994), 413-440.

[25] D.W. Robinson and A. Sikora, Analysis of degenerate elliptic operators of Grušin type, Math. Z. 260(3) (2008), 475-508.

[26] A. Sikora and J. Wright, Imaginary powers of Laplace operators, Proc. Amer. Math. Soc. 129(6) (2001), 1745-1754.

[27] R.S. Strichartz, $L^{p}$ harmonic analysis and Radon transforms on the Heisenberg group, J. Funct. Anal. 96(2) (1991), 350-406.

[28] A.F.M. ter Elst and D.W. Robinson, Spectral estimates for positive Rockland operators, in Algebraic groups and Lie groups, Vol. 9 of Austral. Math. Soc. Lecture Series, 195-213, Cambridge University Press, Cambridge (1997).

[29] S. Thangavelu, Lectures on Hermite and Laguerre expansions, Vol. 42 of Mathematical Notes, Princeton University Press, Princeton, NJ (1993).

[30] J.F. Torres Lopera, The cohomology and geometry of Heisenberg-Reiter nilmanifolds, in Differential geometry, Peñíscola 1985, Vol. 1209 of Lecture Notes in Math., 292-301, Springer, Berlin (1986).

Mathematisches Seminar, Christian-Albrechts-Universität ZU Kiel, Ludewig-MeynStr. 4, D-24118 Kiel, Germany

E-mail address: martini@math.uni-kiel.de

Department of Mathematics, Macquarie University, NSW 2109, Australia

E-mail address: adam.sikora@maths.mq.edu.au 